Provided for non-commercial research and education use. Not for reproduction, distribution or commercial use.

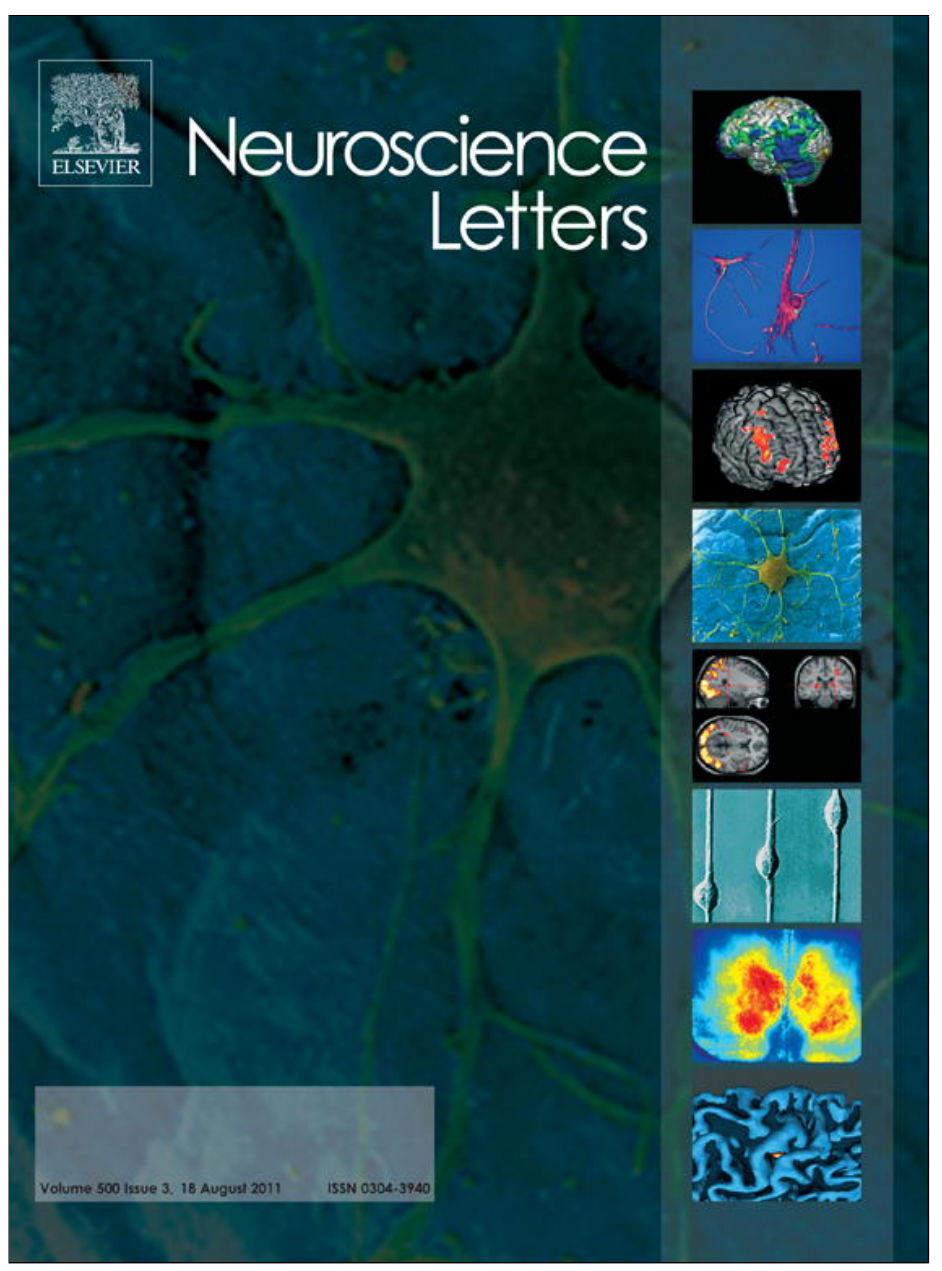

This article appeared in a journal published by Elsevier. The attached copy is furnished to the author for internal non-commercial research and education use, including for instruction at the authors institution and sharing with colleagues.

Other uses, including reproduction and distribution, or selling or licensing copies, or posting to personal, institutional or third party websites are prohibited.

In most cases authors are permitted to post their version of the article (e.g. in Word or Tex form) to their personal website or institutional repository. Authors requiring further information regarding Elsevier's archiving and manuscript policies are encouraged to visit:

http://www.elsevier.com/copyright 


\title{
Insomnia, platelet serotonin and platelet monoamine oxidase in chronic alcoholism
}

\author{
Korona Nenadic Sviglin a,1, Gordana Nedic ${ }^{\mathrm{b}, 1}$, Matea Nikolac ${ }^{\mathrm{b}}$, Maja Mustapic ${ }^{\mathrm{b}}$, \\ Dorotea Muck-Seler ${ }^{b}$, Fran Borovecki ${ }^{c}$, Nela Pivac ${ }^{b, *}$ \\ ${ }^{a}$ Center for Alcoholism and Other Addictions, Psychiatric Hospital Vrapce, Bolnicka cesta 32, HR-1000 Zagreb, Croatia \\ ${ }^{\mathrm{b}}$ Division of Molecular Medicine, Rudjer Boskovic Institute, Bijenicka 54, HR-10000 Zagreb, Croatia \\ ${ }^{c}$ Department for Functional Genomics, Center for Translational and Clinical Research, University of Zagreb School of Medicine, Salata 2 , \\ and University Hospital Center Zagreb, Ksipaticeva 12, HR-10000 Zagreb, Croatia
}

\section{A R T I C L E I N F O}

\section{Article history:}

Received 13 May 2011

Received in revised form 10 June 2011

Accepted 11 June 2011

\section{Keywords:}

Alcoholism

Insomnia

Platelet serotonin

Platelet monoamine oxidase

Smoking

\begin{abstract}
A B S T R A C T
Insomnia is a common sleep disorder frequently occurring in chronic alcoholic patients. Neurobiological basis of insomnia, as well as of alcoholism, is associated with disrupted functions of the main neurotransmitter systems, including the serotonin (5-hydroxytryptamine, 5-HT) system. Blood platelets are considered a limited peripheral model for the central 5-HT neurons, since both platelets and central 5HT synaptosomes have similar dynamics of 5-HT. Platelet 5-HT concentration and platelet monoamine oxidase type B (MAO-B) are assumed to represent biomarkers for particular symptoms and behaviors in psychiatric disorders. The hypothesis of this study was that platelet 5-HT concentration and platelet MAO-B activity will be altered in chronic alcoholic patients with insomnia compared to comparable values in patients without insomnia. The study included 498 subjects: 395 male and 103 female medicationfree patients with alcohol dependence and 502 healthy control subjects: 325 men and 177 women. The effects of early, middle and late insomnia (evaluated using the Hamilton Depression Rating Scale), as well as sex, age and smoking on platelet 5-HT concentration and platelet MAO-B activity were evaluated using one-way ANOVA and multiple regression analysis by the stepwise method. Platelet 5-HT concentration, but not platelet MAO-B activity, was significantly reduced in alcoholic patients with insomnia compared to patients without insomnia. Multiple regression analysis revealed that platelet 5-HT concentration was affected by middle insomnia, smoking and sex, while platelet MAO activity was affected only by sex and age. The present and previous data suggest that platelet 5-HT concentration might be used, after controlling for sex and smoking, as a biomarker for insomnia in alcoholism, PTSD and in rotating shift workers.
\end{abstract}

(c) 2011 Elsevier Ireland Ltd. All rights reserved.
Patients with chronic alcoholism frequently experience sleep disturbances such as insomnia, disrupted sleep, frequent episodes of wakefulness, early wakening, and the reduced quality of life $[6,30]$. Insomnia is present in $36-72 \%$ of alcoholic patients [1], since chronic alcoholism affects sleep quality, and sleep is fragmented and restless [1]. Insomnia can be classified into early, middle and late according to the Hamilton Depression Rating Scale (HDRS) [11]. Neurobiological basis of insomnia, as well as of alcoholism, is associated with disrupted functions of the main

Abbreviations: HDRS, Hamilton Depression Rating Scale; 5-HIAA, 5hydroxyindolacetic acid; MAO-B, monoamine oxidase type B; 5-hydroxytryptamine 5-HT, serotonin; 5-HTTLPR, serotonin transporter polymorphism.

* Corresponding author. Tel.: +3851 4571207; fax: +38514561010.

E-mail address: npivac@irb.hr (N. Pivac).

1 These authors equally contributed to this work. neurotransmitters systems such as serotonin (5-hydroxytryptamine, 5-HT), noradrenalin and histamine. Wakefulness is related to increased 5-HT activity from dorsal raphe [33]. Altered function of the 5-HT system is associated with development of alcoholism, alcohol addiction, alcohol abuse [15,29], and sleep apnea [18], insomnia [34], arousal, sleep-waking states, vigilance behaviors, synchronization of the biological clock and circadian rhythm [34], while sleep disturbances are affected by antidepressants [1]. Insomnia is related to mood instabilities, craving, alcoholic relapse and heavy drinking in chronic alcoholic patients [6]. Prolonged insomnia may lead to self medication with alcohol and chronic alcoholism, while, on the other hand, toxic effects of alcohol in the brain of chronic alcoholics may lead to insomnia [6,30]. Blood platelets are considered as a non-invasive and inexpensive peripheral model for the studies of the central 5-HT neurons, since both platelets and central 5-HT synaptosomes have similar uptake, storage, 
Table 1

Age, platelet 5-HT concentration and platelet MAO-B activity in healthy subjects and alcoholic patients subdivided according to the sex and smoking status.

\begin{tabular}{|c|c|c|c|c|}
\hline & \multicolumn{2}{|l|}{ Men } & \multicolumn{2}{|l|}{ Women } \\
\hline & Smokers & Non-smokers & Smokers & Non-smokers \\
\hline \multicolumn{5}{|l|}{ Age (years) ${ }^{\mathrm{a}}$} \\
\hline Healthy subjects $(N=502)$ & $39.30(10.02) N=95$ & $47.09(13.02) N=230$ & $44.44(8.91) N=39$ & $58.83(17.52) N=138$ \\
\hline Alcoholic patients $(N=498)$ & $48.48(947)^{*} N=238$ & $50.50(10.39) N=157$ & $47.76(9.03) N=58$ & $57.16(12.30) N=45$ \\
\hline \multicolumn{5}{|c|}{ Platelet 5-HT (nmol/mg proteins) $)^{b}$} \\
\hline Healthy subjects $(N=502)$ & $1.09(0.44) N=95$ & $0.94(0.41) N=230$ & $1.14(0.47) N=39$ & $1.10(0.46) N=138$ \\
\hline Alcoholic patients $(N=498)$ & $0.91(0.49)^{*} N=238$ & $0.71(0.41)^{* *} N=157$ & $0.84(0.43)^{* * *} N=58$ & $0.61(0.29)^{* * * *} N=45$ \\
\hline \multicolumn{5}{|c|}{ MAO-B activity (nmol $4 \mathrm{OHQ} / \mathrm{mg}$ proteins/h) ${ }^{\mathrm{c}}$} \\
\hline Healthy subjects $(N=502)$ & $13.13(6.16) N=95$ & $17.00(8.63) N=230$ & $18.60(8.73) N=39$ & $22.63(10.63) N=138$ \\
\hline Alcoholic patients $(N=498)$ & $17.34(9.20)^{*} N=238$ & $18.72(9.86) N=157$ & $20.76(10.75) N=58$ & $24.01(11.76) N=45$ \\
\hline
\end{tabular}

$N$ is the number of subjects.

a $F(7,992)=27.191 ; p<0.001$

b $F(7,992)=15.39 ; p<0.001$.

c $F(7,992)=12.37 ; p<0.001$

$p=0.028-001$ vs. healthy male smokers (one-way ANOVAs followed by Tukey's test).

$p=0.028-0.001$ vs. healthy male non-smokers (one-way ANOVAs followed by Tukey's test).

${ }^{* * *} p=0.001$ vs. healthy female smokers (one-way ANOVAs followed by Tukey's test).

$p=0.019$ vs. healthy female non-smokers (one-way ANOVAs followed by Tukey's test).

release and metabolism of 5-HT [7,12]. Platelet 5-HT concentration has been proposed as a marker of the central serotonergic function $[8,10]$ and a biomarker for particular symptoms and behaviors in psychiatric disorders [35], although this proposal has also been challenged [20]. Platelet monoamine oxidase type $\mathrm{B}$ (MAO-B) has similar biochemical and pharmacological characteristics with the brain MAO-B [22], and was proposed as a marker for the central serotonergic system [22]. Altered platelet MAO-B activity was associated with different psychopathologies and personality traits, vulnerability to drug-abuse, addiction, social mal-adaptation, and dysinhibitory psychopathology [22]. Lower concentration of platelet 5-HT has been demonstrated in chronic alcoholic patients compared to healthy control subjects [23,26,31], while lower [22], as well as unaltered [25] platelet MAO-B activity was found in alcoholic compared to control subjects. Rotating shift workers with sleep disturbances had lower platelet 5-HT concentration compared to day workers [32], while no data evaluating either platelet 5-HT or platelet MAO-B activity and insomnia in alcoholism has been published thus far. Since chronic alcoholics frequently suffer from insomnia [1], and both platelet markers are under the influence of sex and smoking $[21,22]$, the hypothesis of the study was that platelet 5-HT and MAO-B would differ between patients with chronic alcoholism with or without insomnia. The aim of the study was to evaluate platelet 5-HT concentration and platelet MAO-B activity in alcoholic patients subdivided according to the smoking status, sex and presence and/or absence of early, middle or late insomnia.

The study included 498 subjects: 395 male ( 49.5 years old, range 23-77) and 103 female (52.5 years old, range 23-84) medicationfree patients with chronic alcoholism. The diagnosis of alcohol dependency was done using the Structured Clinical Interview for DSM-IV [9] based on DSM-IV criteria [5]. The comparative healthy control group consisted of 502 subjects: 325 male (45.0 years old, range 23-90) and 177 female (55.6 years old, range 23-89) medication-free subjects, with no personal or family history of psychopathology and/or substance abuse or dependence, no hepatic or renal disorders, and alcohol-free for at least $24 \mathrm{~h}$. Patients were admitted into Psychiatric Hospital Vrapce due to the withdrawal syndrome related to alcohol dependency. Since most of the patients were acutely intoxicated with alcohol, the interview and the sampling were done at least $12 \mathrm{~h}$ after the admission at the department, when the patients were sober, with their full cooperation and adequate understanding. Written informed consent was obtained from all subjects, under procedures approved by the
Ethics Committees of the Psychiatric Hospital Vrapce, and University Hospital Center Zagreb, Zagreb, Croatia. Study has been performed in accordance with the ethical standards laid down in the 1964 Declaration of Helsinki. All subjects were subdivided according to the smoking status (into smokers and non-smokers). Alcoholic patients were subdivided according to the items 4 (early insomnia), 5 (middle insomnia) and 6 (late insomnia) of the HDRS [11] into patients without (with score 0 ) or with (i.e. with scores 1-2) early, middle or late insomnia; or without and with insomnia (including early, middle and late insomnia). Blood samples $(8 \mathrm{ml}$ ) were collected into plastic syringes with $2 \mathrm{ml}$ of acid citrate dextrose anticoagulant at $08.00 \mathrm{~h}$. The determination of the platelet 5 -HT concentration was done in platelet rich plasma using spectrofluorimetric method, as previously described [24,27]. Platelet protein concentrations were measured by the method according to Lowry et al. [16]. Platelet MAO-B activity was determined spectrofluorimetrically using kynuramine as a substrate by a slight modification of the method developed by Krajl [14], as previously described [23]. The results, expressed as means (standard deviation), were evaluated using one-way analysis of variance (ANOVA) followed by Tukey's test and multiple regression analysis by the stepwise method (with predictor variables of insomnia (none, early, middle, late), smoking (non-smoker, smoker), sex (male, female), and age). The level of significance was set at $p=0.05$.

Age, platelet 5-HT concentration and platelet MAO-B activity were significantly different (one-way ANOVA and Tukey's test) between alcoholic patients and healthy control subjects subdivided according to the sex and smoking status (Table 1). Male alcoholic smokers were significantly $(p=0.001)$ older than healthy male smokers, while other groups were matched for age. Male $(p=0.028)$ and female $(p=0.019)$ alcoholic smokers had significantly lower platelet 5-HT concentration than healthy male or female smokers, and male or female alcoholic non-smokers had significantly $(p=0.001)$ lower platelet 5-HT values than healthy male or female non-smokers. Platelet MAO-B activity was significantly $(p=0.005)$ higher in male alcoholic smokers compared to male healthy smokers (Table 1 ).

Early (72 and 71\%), middle (68 and 67\%) and late (38 and $41 \%$ ) insomnia occurred at similar frequency in male and female alcoholic patients, respectively (Figs. 1 and 2). To determine the association between the dependent variables such as platelet 5HT concentration (Fig. 1) or platelet MAO-B activity (Fig. 2), and the potential predictor variables (age, sex, smoking status and the presence or absence of early, middle or late insomnia), multiple regression analyses were conducted separately for patients with or 


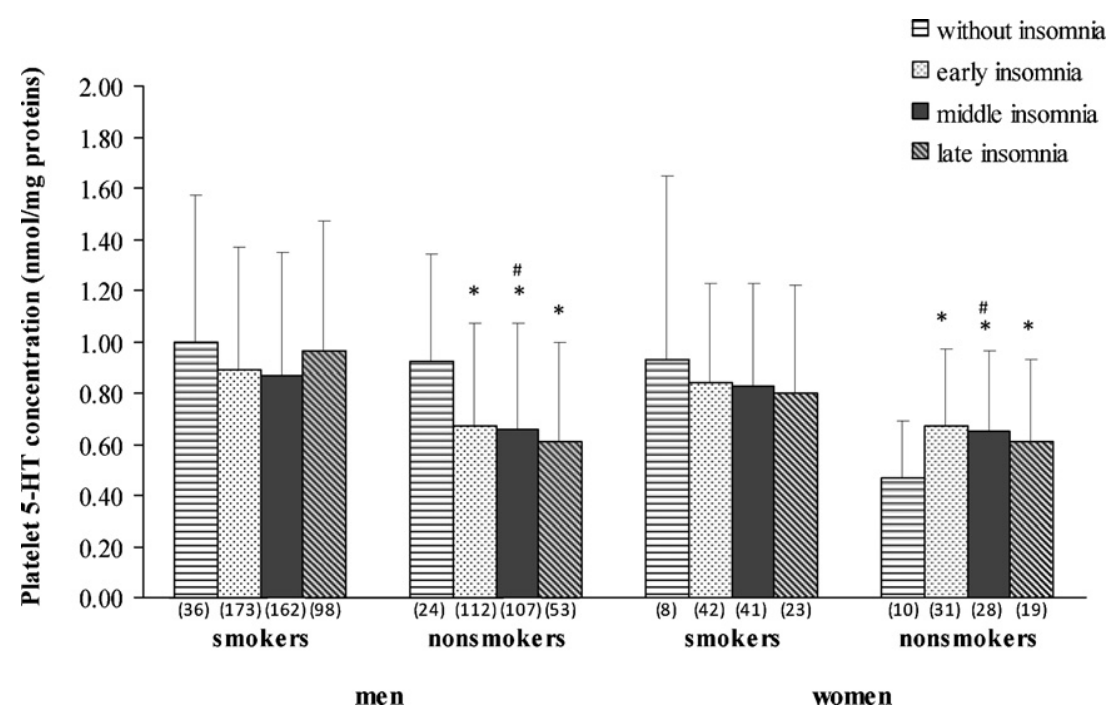

Fig. 1. Platelet $5-\mathrm{HT}$ concentration (mean \pm SD) in alcoholic patients without insomnia and alcoholic patients with early, middle and late insomnia, subdivided according to the sex and smoking status. Numbers in parentheses are the number of subjects. Statistical evaluation of the data is presented in Table $2 .{ }^{*} p=0.001$ vs. the corresponding smokers; ${ }^{* *} p=0.001$ vs. alcoholic patients without insomnia (multiple regression analysis).

without early, middle and late insomnia (Table 2). Multiple regression analyses for platelet 5-HT (significance shown in Fig. 1) as a dependent variable revealed that: (1) smoking status (but not sex, age or early insomnia) significantly $(p=0.001)$ predicted $4.9 \%$ of the variance in platelet 5-HT concentration; (2) smoking status and the presence or absence of middle insomnia (but not sex and age) significantly ( $p=0.032$ ) predicted $5.5 \%$ of the variance in platelet 5-HT concentration, and (3) smoking status and sex (but not age or late insomnia) significantly $(p=0.044)$ predicted $8.8 \%$ of differences in 5-HT values. Multiple regression analyses with platelet MAO-B activity (significance shown in Fig. 2) as a dependent variable showed that: (1) sex and age (but not early insomnia or smoking) significantly predicted $4.4 \%$ of the variance $(p=0.018)$ of the enzyme activity, (2) sex and age (but not middle insomnia or smoking) significantly predicted $5.8 \%$ of the variance $(p=0.038)$ in platelet MAO-B, and (3) sex, but not age, smoking or late insomnia, significantly $(p=0.005)$ predicted $2.9 \%$ variance in platelet MAO-B values (Table 2). Our collective data reveal that platelet 5-HT concentration, but not platelet MAO-B activity, was significantly lower in alcoholic patients with middle insomnia.

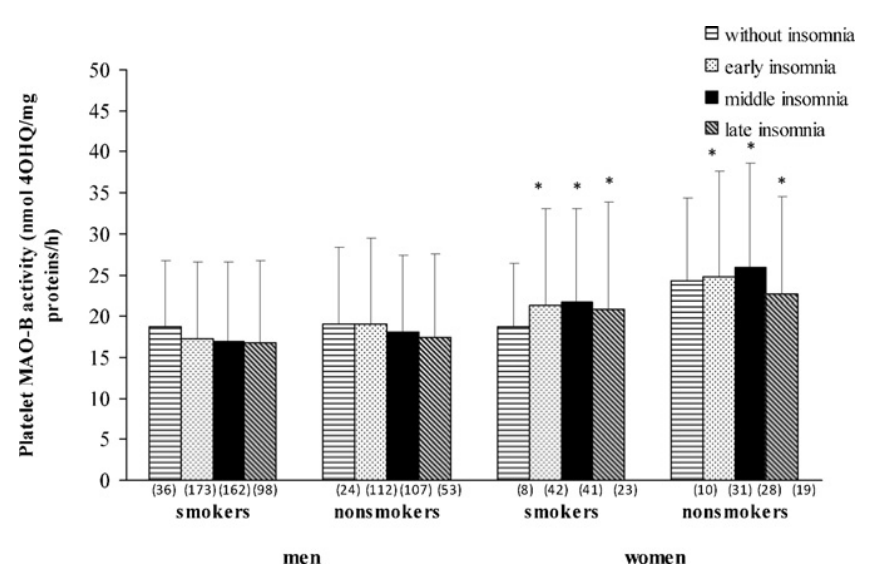

Fig. 2. Platelet MAO-B activity (mean $\pm S D$ ) in alcoholic patients without insomnia and alcoholic patients with early, middle and late insomnia, subdivided according to the sex and smoking status. Numbers in parentheses are the number of subjects. Statistical evaluation of the data is presented in Table $2 .{ }^{*} p=0.005-0.001$ vs. the corresponding male patients (multiple regression analysis).
To further corroborate the significant effect of middle insomnia on dependent variables, multiple regression analyses were used to re-evaluate the influence of the age, sex, smoking status and the presence or absence of insomnia (not subdivided into early, middle or late) on platelet 5-HT concentration or platelet MAOB activity. Analysis confirmed a significant $\left(R^{2}=0.060\right.$; adjusted $\left.R^{2}=0.057 ; F(2,495)=15.888 ; p=0.001\right)$ effect of the presence of insomnia $(t=-2.019 ; p=0.001)$ and smoking $(t=5.315 ; p=0.001)$ on platelet 5-HT concentration. In contrast, platelet MAO-B activity was significantly $\left(R^{2}=0.041\right.$; adjusted $R^{2}=0.037 ; F(2,495)=10.519$; $p=0.001)$ affected by sex $(t=3.690 ; p=0.001)$ and age $(t=2.334$; $p=0.020$ ), but not by presence or absence of insomnia.

Major findings from our study were: (1) alcoholic patients with insomnia (or explicitly with middle insomnia) had significantly lower platelet 5-HT concentration than alcoholic patients without insomnia; (2) the presence of early, middle or late insomnia did not influence platelet MAO-B activity in alcoholic patients; (3) smoking significantly affected platelet 5-HT concentration, and (4) sex and age significantly affected platelet MAO-B activity.

Patients with chronic alcoholism or alcohol abuse frequently (36-72\%) suffer from insomnia $[1,6,30]$. Central 5-HT regulates arousal states, wakefulness, synchronization of the biological clock and circadian rhythm, and alterations of these behaviors may lead to insomnia and sleep disturbances [4]. Platelet 5-HT concentration was lower in rotating shift workers when compared to day workers [32]. We have found lower platelet 5-HT concentration in alcoholic patients with evident middle insomnia (evaluated using HDRS) in comparison to alcoholic patients without middle insomnia. These results were confirmed by the analysis showing that the presence of insomnia (not subdivided into early, middle and late) was significantly associated with platelet 5 -HT concentration. These results are in line with the lower platelet 5-HT values, lower levels of the 5-HT metabolite, 5-hydroxyindolacetic acid (5-HIAA), and higher frequency of the short allele of the serotonin transporter polymorphism (5-HTTLPR) found in workers who work in rotation shifts compared to day workers [32]. The present study did not evaluate the distribution of the 5-HTTLPR variants in alcoholic patients with insomnia, since platelet 5-HT concentration is not affected by the 5-HTTLPR genotypes [27,32]. Although three types of insomnia occurred similarly in male and female patients, our present and previous [28] results show the lack of effect of late insomnia on platelet 5 -HT concentration in alcoholic patients, male or female patients 
Table 2

Results of stepwise multiple regression analysis for variables predicting platelet 5-HT concentration and platelet MAO-B activity.

\begin{tabular}{|c|c|c|c|c|c|}
\hline Variable & $B$ & Std. error & $\beta$ coefficient & $t$ & Sig. \\
\hline \multicolumn{6}{|c|}{ Platelet 5-HT concentration (nmol/mg protein) } \\
\hline \multicolumn{6}{|l|}{ Early insomnia ${ }^{a}$} \\
\hline Constant & 0.692 & 0.034 & & 20.528 & $p<0.001$ \\
\hline Smoking status & 0.207 & 0.044 & 0.221 & 4.730 & $p<0.001$ \\
\hline \multicolumn{6}{|l|}{ Middle insomnia ${ }^{b}$} \\
\hline Constant & 0.785 & 0.057 & & 13.714 & $p<0.001$ \\
\hline Smoking status & 0.201 & 0.045 & 0.213 & 4.459 & $p<0.001$ \\
\hline Middle insomnia & -0.122 & 0.057 & -0.032 & -2.150 & $p<0.001$ \\
\hline \multicolumn{6}{|l|}{ Late insomnia ${ }^{c}$} \\
\hline Constant & 0.706 & 0.050 & & 14.170 & $p<0.001$ \\
\hline Smoking status & 0.263 & 0.059 & 0.260 & 4.437 & $p<0.001$ \\
\hline Gender & -0.141 & 0.070 & -0.119 & -2.023 & $p=0.044$ \\
\hline \multicolumn{6}{|c|}{ Platelet MAO-B activity (nmol 4HOQ/mg protein/h) } \\
\hline \multicolumn{6}{|l|}{ Early insomnia $^{\mathrm{d}}$} \\
\hline Constant & 12.715 & 2.350 & & 5.410 & $p<0.001$ \\
\hline Gender & 4.179 & 1.182 & 0.167 & 3.536 & $p<0.001$ \\
\hline Age & 0.110 & 0.047 & 0.122 & 2.371 & $p=0.018$ \\
\hline \multicolumn{6}{|l|}{ Middle insomnia ${ }^{e}$} \\
\hline Constant & 12.743 & 2.396 & & 5.317 & $p<0.001$ \\
\hline Gender & 5.153 & 1.193 & 0.208 & 4.320 & $p<0.001$ \\
\hline Age & 0.099 & 0.047 & 0.100 & 2.081 & $p=0.038$ \\
\hline \multicolumn{6}{|l|}{ Late insomnia ${ }^{\mathrm{f}}$} \\
\hline Constant & 17.567 & 0.689 & & 25.482 & $p<0.001$ \\
\hline Gender & 4.139 & 1.465 & 0.170 & 2.825 & $p=0.005$ \\
\hline
\end{tabular}

From the independent variables calculated in this regression (age, sex, smoking status and the presence or absence of early, middle or late insomnia), those listed in table were significantly associated with platelet 5-HT concentration and platelet MAO activity at the indicated level of significance in the model.

a Fit of the model: $R^{2}=0.049$; adjusted $R^{2}=0.047 ; \mathrm{df}=1,434 ; F=22.370 ; p<0.001$.

b Fit of the model: $R^{2}=0.055 ;$ adjusted $R^{2}=0.050 ; \mathrm{df}=1,413 ; F=4.624 ; p=0.032$.

c Fit of the model: $R^{2}=.088$; adjusted $R^{2}=.081 ; \mathrm{df}=1,268 ; F=4.093 ; p=0.044$.

d Fit of the model: $R^{2}=0.044 ;$ adjusted $R^{2}=0.040 ; \mathrm{df}=1,433 ; F=5.621 ; p=0.018$.

e Fit of the model: $R^{2}=0.058$; adjusted $R^{2}=0.053 ; \mathrm{df}=1,413 ; F=4.331 ; p=0.038$

f Fit of the model: $R^{2}=.029 ;$ adjusted $R^{2}=.025 ; \mathrm{df}=1,269 ; F=7.982 ; p=0.005$.

on dialysis with chronic renal failure [24], or in male veterans with posttraumatic stress disorder (PTSD) [19]. The differences between the aforementioned studies were that these subjects were not categorized according to the smoking status. Our results concur with our preliminary data showing lower platelet 5-HT concentration in the smaller number of male alcoholics with middle insomnia (who were not subdivided according to the smoking status), and in male PTSD veterans with insomnia and flashbacks [28], compared to groups without sleep disturbances. Platelet 5-HT concentration was not affected by medication, age, comorbid diagnoses or suicidal attempts, since the present study included medication-free alcoholic patients; the effect of age was not significant; and comorbid psychiatric disorders or suicide attempts were reported to be devoid of any significant effect on platelet 5-HT concentration in male or female alcoholic patients [21]. The frequency of smoking was similar between male (60\%) and female (56\%) alcoholics. The finding that male alcoholic smokers have higher platelet 5HT concentration than male alcoholic non-smokers agrees with previously published data $[21,31]$. The lack of clear sex related differences in platelet 5-HT concentration is in contrast with some previous data, presumably due to the fact that alcoholic patients were not subdivided according to the smoking status $[23,26,28]$, but this finding is in line with the previous studies after subdivision according to the smoking status [21]. Reduced platelet 5-HT concentration was found in alcoholic subjects compared to healthy control subjects $[21,23,26]$; but in addition, the present study confirmed significantly lower platelet 5-HT values in male and female alcoholic smokers and non-smokers compared to corresponding healthy male and female smokers and nonsmokers. This finding stresses the need of subdividing data according to the smoking status, since smoking significantly affected platelet 5 -HT values in male and female alcoholic patients with or without insomnia.
In line with our previous preliminary findings showing the lack of effect of various sleep disturbances on platelet MAO activity in much smaller groups of chronic alcoholic patients, combat exposed veterans with PTSD, or patients with major depressive disorder [28], we have found that the presence of early, middle or late insomnia did not change platelet MAO-B activity in male or female alcoholic patients. In contrast to our present and previously published data [28], higher platelet MAO-B activity was found in depressed patients with late insomnia [2], and in alcoholics during the recovery period after withdrawal from heavy alcohol abuse with fatigue, insomnia and reduced sleep [3] compared to corresponding controls. However, the data on the smoking status were not reported in these older studies. Platelet MAO-B activity was significantly affected by sex and age, as expected [22], but not by smoking, although the substances from the cigarette smoke reduce platelet MAO-B activity [22,31]. Platelet MAO-B activity was similar between alcoholic patients and healthy subjects, higher in male alcoholic smokers when compared to healthy male smokers, but was not significantly affected by insomnia, medication (present study), or comorbid psychiatric diagnoses [23].

The reduced prefrontal 5-HT activity was associated with disturbed sleep-wakefulness rhythm and loss of emotional control [13], while maximal 5-HT activity is achieved during waking [33]. Low central 5-HT activity is related to development of aggressive and impulsive behavior [17], that frequently occurs in chronic alcoholic patients. Although reductions in platelet 5-HT concentration, decreases in 5-HIAA levels and over-expression of the short allele of the 5-HTTLPR polymorphism [32] were found in rotation shift workers (who have disturbed sleep patterns) compared to daily workers, our present data do not confirm the hypothesis that platelet MAO-B activity (proposed to reflect the activity of the central 5-HT system [22]), might be used as a peripheral biomarker 
of the insomnia. In contrast, although platelet 5-HT concentration does not reflect central 5-HT activity, platelet 5-HT concentration was significantly and inversely associated with middle insomnia in alcoholic patients.

Present and previous results $[28,32]$ suggest that platelet 5-HT concentration might be used, after controlling for smoking and sex, as a peripheral biomarker for insomnia in alcoholism, PTSD and in rotating shift workers.

\section{Acknowledgments}

This work was supported by Croatian Ministry of Science, Education and Sport, grants numbers 098-0982522-2455, 0980982522-2457 and 108-1081874-1923.

\section{References}

[1] V.C. Abad, C. Guilleminault, Sleep and psychiatry, Dialogues Clin. Neurosci. 7 (2005) 291-303.

[2] H. Agren, L. Oreland, Early morning awakening in unipolar depressives with higher levels of platelet MAO activity, Psychiatry Res. 7 (1982) 245-254.

[3] C. Alling, J. Balldin, K. Bokstrom, C.G. Gottfries, I. Karlsson, G. Langstrom, Studies on duration of a late recovery period after chronic abuse of ethanol - a crosssectional study of biochemical and psychiatric indicators, Acta Psychiatr. Scand. 66 (1982) 384-397.

[4] R. Andretic, P. Franken, M. Tafti, Genetics of sleep, Annu. Rev. Genet. 42 (2008) 361-388.

[5] American Psychiatric Association, Diagnostic and Statistical Manual of Mental Disorders, fourth ed., American Psychiatric Press, Washington, DC, 1994.

[6] K.J. Brower, Insomnia, alcoholism and relapse, Sleep Med. Rev. 7 (2003) 523-539.

[7] A. Camacho, J.E. Dimsdale, Platelets and psychiatry: lessons learned from old and new studies, Psychosom. Med. 62 (2000) 326-336.

[8] E. Emanuele, N. Brondino, M. Bertona, S. Re, D. Geroldi, Relationship between platelet serotonin content and rejections of unfair offers in the ultimatum game, Neurosci. Lett. 437 (2008) 158-161.

[9] M. First, R. Spitzer, J. Williams, M. Gibbon, Structured Clinical Interview for DSM-IV Axis I Disorders (SCID-I). Handbook of Psychiatric Measures, first ed., American Psychiatric Association, Washington, DC, USA, 2000, pp. 49-53.

[10] R. Gregory, D.O. Lande, Whole blood serotonin levels among pretrial murder defendants, J. Psychiatry Law 31 (2003) 287-303.

[11] M. Hamilton, A rating scale for depression, J. Neurol. Neurosurg. Psychiatry 23 (1960) 56-62.

[12] P.D. Hrdina, Platelet serotonergic markers in psychiatric disorders: use, abuse and limitations, J. Psychiatry Neurosci. 19 (87-88) (1994) 90.

[13] J. Kohyama, Sleep health and asynchronization, Brain Dev. 33 (2011) 252-259.

[14] M. Krajl, A rapid microfluorimetric determination of monoamine oxidase Biochem. Pharmacol. 14 (1965) 1684-1686.

[15] D.M. Lovinger, Serotonin's role in alcohol's effects on the brain, Alcohol Health Res. World 21 (1997) 114-120.
[16] O.H. Lowry, N.J. Rosebrough, A.L. Farr, R.J. Randall, Protein measurement with the Folin phenol reagent, J. Biol. Chem. 193 (1951) 265-275.

[17] I. Lucki, The spectrum of behaviors influenced by serotonin, Biol. Psychiatry 44 (1998) 151-162.

[18] U.D. McCann, F.P. Sgambati, A.R. Schwartz, G.A. Ricaurte, Sleep apnea in young abstinent recreational MDMA ("ecstasy") consumers, Neurology 73 (2009) 2011-2017.

[19] D. Muck-Seler, N. Pivac, M. Jakovljevic, M. Sagud, A. Mihaljevic-Peles, Platelet 5 -HT concentration and comorbid depression in war veterans with and without posttraumatic stress disorder, J. Affect. Disord. 75 (2003) 171-179.

[20] B. Mueller-Oerlinghausen, J. Roggenbach, L. Franke, Serotonergic platelet mark ers of suicidal behavior - do they really exist? J. Affect. Disord. 79 (2004) 13-24.

[21] K. Nenadic-Sviglin, G. Nedic, M. Nikolac, D. Kozaric-Kovacic, T. Stipcevic, D. Muck Seler, N. Pivac, Suicide attempt, smoking, comorbid depression, and platelet serotonin in alcohol dependence, Alcohol 45 (2011) 209-216.

[22] L. Oreland, Platelet monoamine oxidase, personality and alcoholism: the rise, fall and resurrection, Neurotoxicology 25 (2004) 79-89.

[23] N. Pivac, D. Kozaric-Kovacic, M. Mustapic, M. Dezeljin, K. NenadicSviglin, D. Muck-Seler, New research on alcohol abuse and alcoholism (peripheral biological markers in alcoholism), in: T. Walcott (Ed.), Drug and Alcohol Abuse Research Focus, Nova Publishers, NY, USA, 2007, pp. 1-62.

[24] N. Pivac, D. Muck-Seler, I. Barisic, M. Jakovljevic, Z. Puretic, Platelet serotonin concentration in dialysis patients with somatic symptoms of depression, Life Sci. 68 (2001) 2423-2433.

[25] N. Pivac, D. Muck-Seler, D. Kozaric-Kovacic, M. Mustapic, K. Nenadic-Sviglin, M. Dezeljin, Platelet monoamine oxidase in alcoholism, Psychopharmacology (Berl) 182 (2005) 194-196.

[26] N. Pivac, D. Muck-Seler, M. Mustapic, K. Nenadic-Sviglin, D. Kozaric-Kovacic Platelet serotonin concentration in alcoholic subjects, Life Sci. 76 (2004) 521-531.

[27] N. Pivac, G. Nedic, M. Mustapic, A. Babic, T. Stipcevic, F. Borovecki, S. Hajnsek, D. Muck-Seler, The lack of genotype-phenotype relationship between platelet serotonin concentration and serotonin transporter gene promoter polymorphism in healthy subjects, Neurosci. Lett. 462 (2009) 45-48.

[28] N. Pivac, G. Nedic, M. Nikolac, K. Nenadic-Sviglin, D. Kozaric-Kovacic, M. Mustapic, Z. Kovacic, M. Grubisic Ilic, F. Borovecki, S. Hajnsek, D. Muck-Seler, Sleep disturbances and serotonergic markers in psychiatric disorders, in: A.M Columbus (Ed.), Advances in Psychology Research, Nova Science Publishers, NY, USA, 2010, pp. 1-85.

[29] J.E. Ratsma, O. Van Der Stelt, W.B. Gunning, Neurochemical markers of alcoholism vulnerability in humans, Alcohol Alcohol. 37 (2002) 522-533.

[30] T. Roehrs, T. Roth, Sleep, sleepiness, sleep disorders and alcohol use and abuse, Sleep Med. Rev. 5 (2001) 287-297.

[31] L.G. Schmidt, P. Dufeu, A. Heinz, S. Kuhn, H. Rommelspacher, Serotonergic dysfunction in addiction: effects of alcohol, cigarette smoking and heroin on platelet 5-HT content, Psychiatry Res. 72 (1997) 177-185.

[32] S. Sookoian, C. Gemma, T.F. Gianotti, A. Burgueno, A. Alvarez, C.D. Gonzalez, C.J. Pirola, Serotonin and serotonin transporter gene variant in rotating shift workers, Sleep 30 (2007) 1049-1053.

[33] L. Staner, Sleep disturbances, psychiatric disorders, and psychotropic drugs, Dialogues Clin. Neurosci. 7 (2005) 323-334.

[34] R. Ursin, Serotonin and sleep, Sleep Med. Rev. 6 (2002) 55-69.

[35] L. Zhang, H. Li, D. Benedek, X. Li, R. Ursano, A strategy for the development of biomarker tests for PTSD, Med. Hypotheses 73 (2009) 404-409. 